\title{
GENERATIVE MECHANISMS OF A SURVIVAL TIME: THE CASE OF THE INVERSE WEIBULL MODEL
}

\section{PASQUALE ERTO, ANTONIO LEPORE and BIAGIO PALUMBO}

\author{
University of Naples Federico II \\ Naples \\ Italy \\ e-mail: bpalumbo@unina.it
}

\begin{abstract}
When only a small sample is available, it is well known that the empirical distribution contains scarce information about the shape of the hazard function as well as about the far-right tail of the "parent" probability density. So, the probabilistic analysis of the mechanisms that lead to the involved random variable can be a decisive factor to identify the appropriate distribution model. In this context, three recurrent degradation mechanisms ("simple degradation", "stress-strength", and "latent-defensive-attempts") which lead exactly to the inverse Weibull survival time are identified and mathematically formulated. They are derived as first passage time distributions. Then, the proposed mechanism approach, applied to the proper identification of this relatively unknown survival model, is illustrated by means of two real world examples.
\end{abstract}

\section{Introduction}

It is well-known that individuals, even belonging to the same population, vary in their susceptibilities to potential hazards and that such variability reflects intrinsic heterogeneity as well as differences in 2010 Mathematics Subject Classification: 62.

Keywords and phrases: disease modelling, damage accumulation, survival analysis, hazard rate, mean residual life.

Received June 17, 2016

(c) 2016 Scientific Advances Publishers 
exposures. In these cases, the unimodal shape of the hazard rate is found and explained as suggested in (Aalen [1]). Specifically, the high-risk individuals die at beginning of the life time $t$ and give rise to a rising hazard rate. Then, the hazard rate starts to decline because only the lowrisk individuals survive. For instance, sometimes this happens for cancer patients, when the longer the patients live beyond a certain time, the higher are their chances to survive. In general, the intrinsic human heterogeneity that gives rise to their different susceptibilities to toxicants is proved, as instance, by observations that: not all workers exposed to benzidine develop urinary bladder cancer; not all humans are equally susceptible to air pollutants; the individual-to-individual variability in disease susceptibility is associated with a variety of genetic factors (Olin et al. [25]).

In general, sample sizes in excess of 200-300 are required to reliably distinguish between distributional forms of the survival time (see, e.g., Haas [13]). Viceversa, when only small samples are available, the knowledge of the main mechanisms that lead to a specific survival time can supplement or even prevail over the usual statistical procedures exploited to identify the corresponding distribution model (Baker [3]; Slud \& Suntornchost [26]). To be effective, these mechanisms must represent different possible interactions between the above discussed intrinsic heterogeneity and differences in exposures. Moreover, they can support the degradation analysis and monitoring as shown by $\mathrm{Lu}$ and Meeker [22] and by Gebraeel et al. [9]. In fact, in absence of observed injuries or failures, degradation analysis is an alternate approach that uses a sequence of degradation measures - suggested by a degradation model - to assess the lifetime distribution (Bian \& Gebraeel [5]).

In this context, the paper provides the mathematical models of three typical generative mechanisms, of the inverse Weibull random variable, found in biometry and reliability: "simple degradation", "stress-strength", and "latent-defensive-attempts". So, the paper helps exploiting the inverse Weibull model to give correct answers for these specific hazard problems, for which it is the natural interpretative stochastic model. 
For the proposed degradation mechanisms, the inverse Weibull model is derived as first passage time distribution function. The used approach is along the lines shown by Ballani et al. [4] and Glen [11] in their stimulating papers.

Doubtless, the inverse Weibull random variable is not as widely known as the Weibull, for instance, and so it is seldom identified. Moreover, the inverse Weibull model is referred to by many different names like "complementary Weibull" (Drapella [7]), "reciprocal Weibull" (Lu \& Meeker [22]; Mudholkar \& Kollia [23]), "Fréchet-type" (Johnson et al. [16]; Harlow [14]), and "inverse Weibull" (Johnson et al. [15]; Murthy et al. [24]).

Obviously, the inverse of the inverse Weibull data follows a Weibull distribution. So the parameter estimates of the inverse Weibull distribution can be easily obtained by applying to its reciprocal data the same standard procedures implemented in packages for the Weibull model (Murthy et al. [24]).

The remainder of the paper is organized as follows. The next section gives a brief summary of the peculiar statistical properties of the inverse Weibull model. Section 3 shows the three typical models of mechanisms of failure that lead exactly to the inverse Weibull distribution. In Section 4 , the inverse Weibull model is compared with other commonly-known distributions and it is shown that a pure empirical fitting of a model to the inverse Weibull data can lead to wrong prognoses. In Section 5, two classical discriminant criteria and their probability of success are discussed. Then, two applicative examples to demonstrate the use of the proposed generative mechanisms - when only tiny data sets are available - are shown in Section 6.

\section{Statistical Properties}

The probability density function of the inverse Weibull random variable $T$, with scale parameter $a$ and shape parameter $b$, is (see Figure 1):

$$
f(t)=a b(a t)^{-(b+1)} \exp \left\{-(a t)^{-b}\right\}, \quad t \geq 0, \quad a, b>0 .
$$


It is skewed and unimodal with mode at $t_{m}=\{b /(b+1)\}^{1 / b} / a$. The $k$-th moment of the inverse Weibull random variable is $E\left\{T^{k}\right\}=1 / a^{k} \Gamma(1-k / b)$ and it exists if $b>k$. Then the mean $E\{T\}=(1 / a) \Gamma(1-1 / b)$ and the variance $\operatorname{Var}\{T\}=\left(1 / a^{2}\right)\left\{\Gamma(1-2 / b)-\Gamma^{2}(1-1 / b)\right\}$ follow.

The most distinctive feature of the inverse Weibull model is its heavy right tail, being its probability density infinitesimal of lower order than the negative exponential as $t$ goes to infinity.

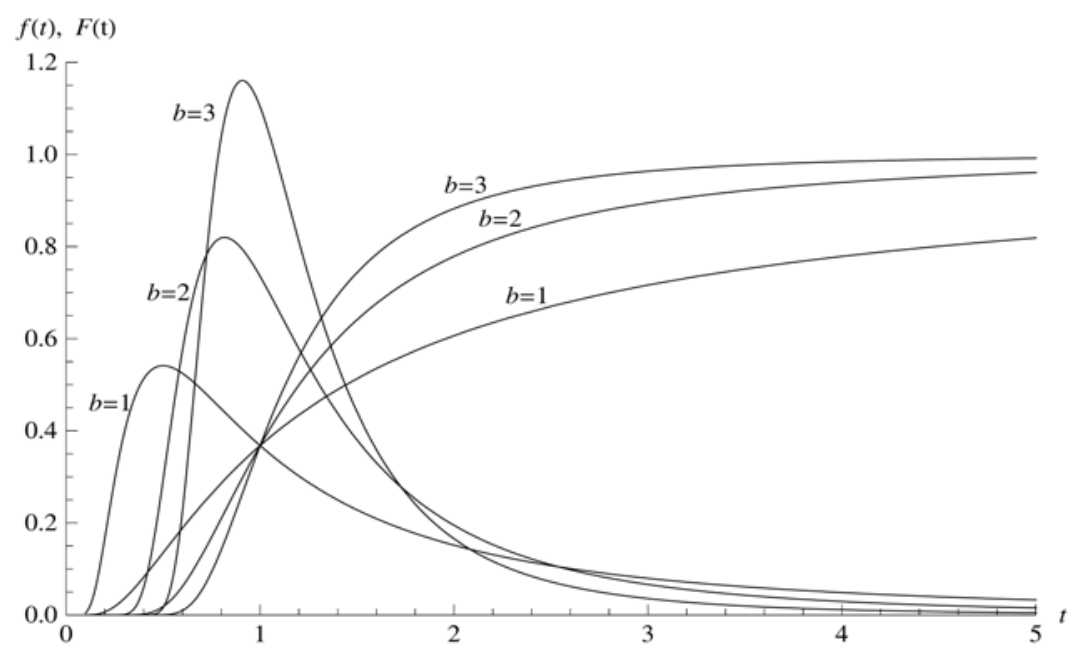

Figure 1. Probability density (ogive-shaped) and distribution functions (S-shaped) of the inverse Weibull model ( $a=1, b=1,2,3)$.

The distribution function $F(t)$ (see Figure 1), the survival function $R(t)$, and the hazard rate $h(t)$ (see Figure 2) are easily derived from (1):

$$
\begin{gathered}
F(t)=1-R(t)=P(T \leq t)=\int_{0}^{t} f(x) d x=\exp \left\{-(a t)^{-b}\right\}, \\
h(t)=\frac{f(t)}{R(t)}=\frac{a b(a t)^{-(b+1)}}{\exp \left\{(a t)^{-b}\right\}-1}, \quad t>0 .
\end{gathered}
$$


The hazard rate is infinitesimal as $t$ goes to infinity. It is unimodal and belongs to the UBT (upside-down bathtub shaped hazard) class (Glaser [10]) with only one change point. We found that the abscissa of the change point is (Figure 2):

$$
t_{m}=\frac{1}{a}\left[b\left\{1+b+b W\left(-\frac{1}{b}(1+b) \exp (-1-1 / b)\right)\right\}^{-1}\right]^{1 / b}
$$

where $W(\cdot)$ is the Lambert function (i.e., the "product logarithm" function for any argument value in the interval $[-1 / e, \infty))$. It can be easily shown that an algebraic approximation of this important abscissa is (Figure 2):

$$
\tilde{t}_{m}=\frac{b^{1 / b}}{2 a}\left[1+(1+b)^{-1 / b}\right]
$$

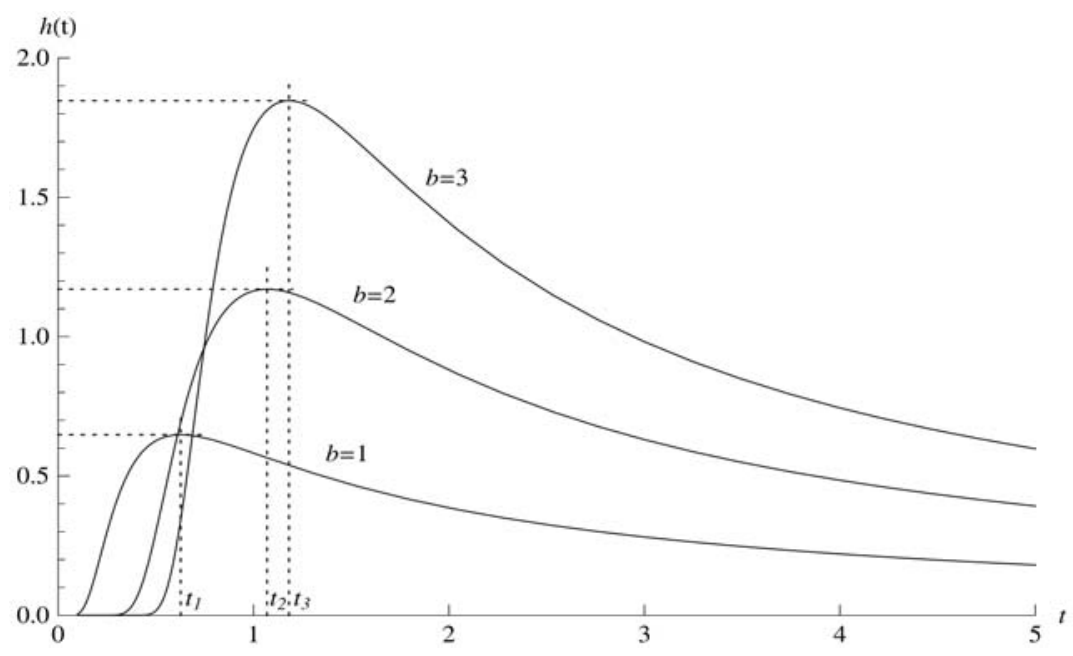

Figure 2. Hazard rates of the inverse Weibull model for $b=1,2,3$ $(a=1)$ and abscissas $t_{1}=0.627, t_{2}=1.07, t_{3}=1.18$ of the respective global maxima (the approximate formula (5) gives: $\widetilde{t}_{1}=0.750, \widetilde{t}_{2}=1.12$, $\left.\tilde{t}_{3}=1.18\right)$. 
The mean residual life $\left(\mathrm{MRL}_{R}\right.$, also called the life expectancy of the $R$ fraction of items lived longer than $t_{R}$ ) is

$$
m\left(t_{R}\right)=\frac{1}{R\left(t_{R}\right)} \int_{t_{R}}^{+\infty} x f(x) d x-t_{R}=\frac{1 / a \Gamma\left(1-1 / b, a^{-b} t_{R}^{-b}\right)}{1-\exp \left\{-\left(a t_{R}\right)^{-b}\right\}}-t_{R}, \quad b>1,
$$

being $\Gamma\left(1-1 / b, a^{-b} t^{-b}\right)$ the lower incomplete gamma function. The $\mathrm{MRL}_{R}$ function of the inverse Weibull model is bathtub-shaped. The $\mathrm{MRL}_{R}$ decreases from the initial value $E(T)$ (as $t$ goes to 0 ) to its minimum at the change point $t_{0}$ and then increases infinitely as $t$ goes to infinity. Being $d m(t) / d t=m(t) h(t)-1$ (e.g., see Lai \& Xie [17], Chapter 4), the change point $t_{0}$ must necessarily solve the equation $m(t) h(t)=1$. In practice, this peculiar $\mathrm{MRL}_{R}$ shape can be found, for example, in some biometry problems when the longer the patient's survival time from his tumor ablation the better his prognosis.

\section{Physical Models of Mechanisms of Failure}

\section{1. "Simple degradation" mechanism}

Let $Y(t)$ be a system damage accumulation index which is modelled by a non-decreasing and right-continuous stochastic process on the range $[0, \infty)$ of the run time $t$. If $D$ is the threshold (maximum, positive) value for $Y(t)$, then, the first passage time $T$ of $Y(t)$ over $D$ has distribution function $F(t)=P(T \leq t)=P\{Y(t) \geq D\}$. Let, for every $t$, the distribution function of $Y(t)$ be reasonably fitted by a Weibull model with constant shape parameter and scale parameter $u$, function of $t$, modelled by a generic power law:

$$
u(t)=k t^{h} ; \quad t, h, k>0
$$


then, the life-to-failure of the system has the inverse Weibull distribution function. In fact, consider a Weibull random variable $Y(t)$ with probability density function:

$$
g(y)=v / u(t)\{y / u(t)\}^{v-1} \exp \left[-\{y / u(t)\}^{v}\right] ; \quad y \geq 0, \quad v, u>0,
$$

where $v$, the shape parameter, is constant (since characteristic of the involved phenomenon) and $u(t)$, the scale parameter, is the drift function (7). The first passage time $T$ of $Y(t)$ over $D$ has distribution function:

$$
F(t)=P\{Y(t) \geq D\}=1-\int_{0}^{D} g(y) d y=\exp \left[-\{D / u(t)\}^{v}\right] .
$$

Substituting $u(t)=k t^{h}$ back into the previous relationship, we obtain:

$$
F(t)=\exp \left[-\left\{(k / D)^{1 / h} t\right\}^{-v h}\right]
$$

and, on setting $a=(k / D)^{1 / h}$ and $b=v h$, the inverse Weibull distribution function follows.

This mechanism is found in many prosthesis corrosion phenomena that give rise to failures only when they reach a threshold deepness $D$. The mechanism is found also in many biologic degenerative phenomena (i.e., gradual degradation of organs and cells) where the loss of function appears when the degradation level $Y(t)$ reaches a threshold value. Besides, this mechanism is found when tumors spread potential metastases with a dissemination probability proportional to their size $Y(t)$. Hence, a tumor size greater than a given threshold value $D$ causes a rate of occurrence of metastases which is really first increasing and then decreasing (see Le Cam \& Neyman [19], p. 253) like the inverse Weibull one.

It should be mentioned that a linear function of $t$ degradation index, $Y(t)$, that gives rise to an inverse Gaussian distribution, has been considered by Lee and Tang ([21]) that analogously defined the failure time as first-passage of $Y(t)$ over a constant threshold. 


\section{2. "Stress-strength" mechanism}

Let the stress $S(t)$ (in the broad sense) be modelled by a stationary and right continuous stochastic process on the range $[0, \infty)$ of the run time $t$. Let the distribution function of $S(t)$ be reasonably fitted by a Weibull distribution independent of $t$. Let the strength $Z(t)$, that opposes $S(t)$, be a decreasing function of time $t$ that can be modelled by a generic power law:

$$
Z(t)=k t^{-h} ; \quad t, h, k>0
$$

Then, the first passage time $T$ of $S(t)$ over $Z(t)$ has distribution function $F(t)=P(T \leq t)=P\{S(t) \geq Z(t)\}$ of inverse Weibull type. In fact, if, for every $t$, the stress $S(t)$ is a Weibull random variable with probability density function:

$$
g(s)=v / u(s / u)^{v-1} \exp \left\{-(s / u)^{v}\right\}, \quad s \geq 0, \quad u, v>0,
$$

it follows:

$$
F(t)=P\{S(t) \geq Z(t)\}=1-\int_{0}^{Z(t)} g(s) d s=\exp \left[-\{Z(t) / u\}^{v}\right] .
$$

By substituting $Z(t)=k t^{-h}$ back into the previous relationship, it follows:

$$
F(t)=\exp \left[-\left\{(u / h)^{1 / h} t\right\}^{-v h}\right]
$$

and by renaming $a=(u / h)^{1 / h}$ and $b=v h$, the inverse Weibull distribution is obtained.

This mechanism is found in patients with a decreasing vital strength following (11) (e.g., because they are subject to intensive and prolonged chemotherapy) and subject to a cancer having a random virulence or gravity $S$. In these cases, an hazard rate which increases first quickly and then decreases gradually, is sometimes surprisingly observed (see 
Carter et al. [6], p. 79). The decline of the hazard rate depends on the fact that, for some individuals, the probability $P\{S(t) \geq Z(t)\}$ is low because the aggressiveness of the carcinoma is constantly low (12). That causes a sort of natural selection of the patients that explains the peculiar shape of the mean residual life function (6) and complies with the above empirical observation that the longer the patients live the higher are their chances to survive.

\section{3. "Latent-defensive-attempts" mechanism}

Suppose that a system is subject to a life attack (e.g., a disease or failure) $\Phi$ and the physiological latent defensive attempt $\Delta$ is repeated according to the homogeneous Poisson process on $[0, \infty)$ with intensity function $\beta$. Let $(\Delta>\Phi)$ be the event "success of a defensive attempt," that is the exceedance of $\Delta$ over $\Phi$. If the probability $P_{S}(t)=P(\Delta>\Phi)$ depends on the incubation time $t$ (but not on the number of previously occurred defensive actions) according to a generic power law decreasing function:

$$
P_{S}(t)=k t^{-h} ; \quad h>1, \quad k>0, \quad t \geq k^{\frac{1}{h}}
$$

then, the exceedance time $T$, of $\Delta$ over $\Phi$, has survival function $R(t)=P(T>t)=P\{\Delta \cap(\Delta>\Phi)\}$ of inverse Weibull type. In fact, suppose that the random variable $N_{a}$, that counts the physiological defensive attempts $\Delta$ against the disease/failure $\Phi$, follows the Poisson law:

$$
\begin{gathered}
P\left(N_{a}=n_{a}\right)=\left\{(\beta t)^{n_{a}} / n_{a} !\right\} \exp (-\beta t) ; \\
n_{a}=0,1,2, \ldots, \quad \beta>0,
\end{gathered}
$$

then, the probability of survival to time $t$ is:

$$
\begin{aligned}
R(t)= & \beta t \exp (-\beta t)\left(k t^{-h}\right)+\frac{(\beta t)^{2}}{2 !} \exp (-\beta t)\left\{1-\left(1-k t^{-h}\right)^{2}\right\} \\
& +\frac{(\beta t)^{3}}{3 !} \exp (-\beta t)\left\{1-\left(1-k t^{-h}\right)^{3}\right\}+\cdots=1-\exp \left(-\beta k t^{-(h-1)}\right) .
\end{aligned}
$$


This equation expresses simply that the survival implies one successful defensive attempt, or two defensive attempts not both unsuccessful, or three defensive attempts not all unsuccessful, and so on. Then, on setting $b=h-1$ and $a=(\beta k)^{-1 / b}$, the inverse Weibull survival function follows.

This mechanism is found when the immune system works randomly against antigens, and its effectiveness decreases as the disease lasts (see Le Cam \& Neyman [19], p. 15). An example of this mechanism is reported in (Lee [20]) where the survival times of insects exposed to a new insecticide are studied. In this case, the effectiveness of the immune response of the insects decreases very slowly over time. Moreover, the lack of memory of the immune system of insects, discussed in (Vilmos \& Kurucz [29]), allows modelling their defensive attempts, against the toxic substance, according to the homogeneous Poisson process.

\section{Comparing the Inverse Weibull with other Commonly-known Distributions}

In order to better understand the peculiarities of the inverse Weibull model and to compare them with those of the other commonly-known distributions, the chart proposed in (Glen [11]; Vargo et al. [28]) can be very useful. In Figure 3, this chart is drawn including the inverse Weibull together with the other few models having upside-down bathtub (UBT) shaped hazard function. 


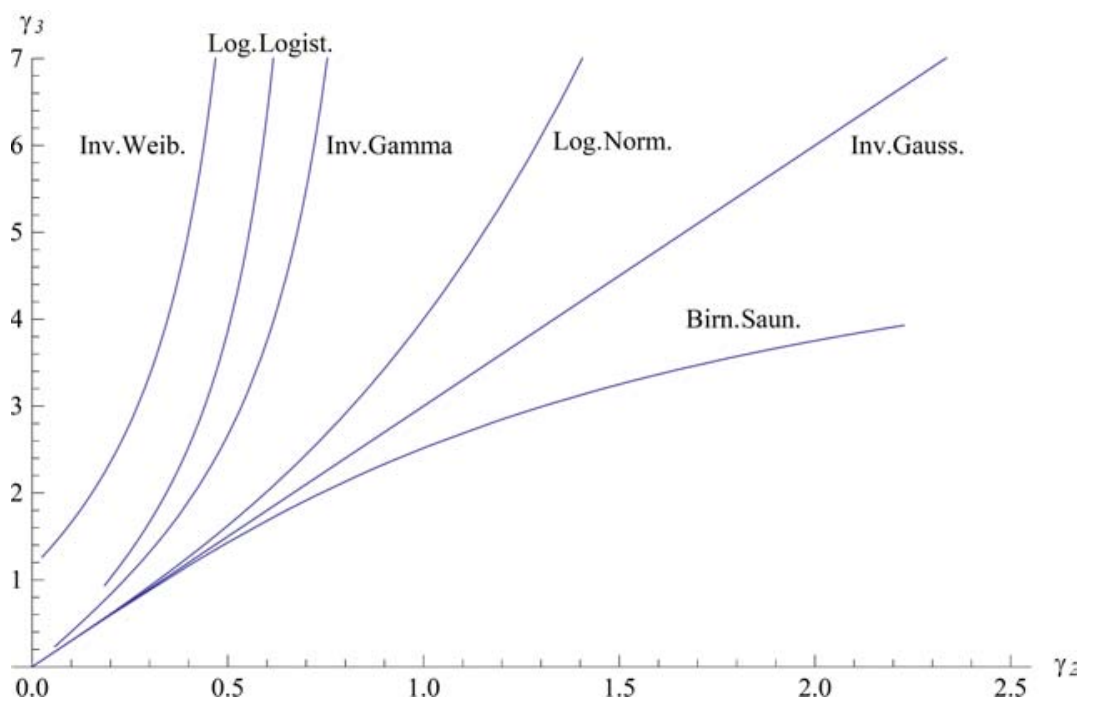

Figure 3. Coefficient of variation $\gamma_{2}$ against skewness $\gamma_{3}$ for various hazard models.

In this chart, the coefficient of variation $\gamma_{2}=\sigma / \mu$ is plotted against skewness $\gamma_{3}=E\left\{(X-\mu)^{3}\right\} / \sigma^{3}$ for five alternative distribution models. Skewness is used to comparatively measure the tendency for one of their tails to be heavier than the other. The plot usually includes all possible pairs $\left(\gamma_{2}, \gamma_{3}\right)$ that a model can attain. The set of values that the inverse Weibull $\left(\gamma_{2}, \gamma_{3}\right)$ pairs can assume fall to the left of those of all the other models, helping to fill a gap on the extreme left of the chart. Since it occupies a small part of the chart, the inverse Weibull model confirms the fact that only peculiar data, corresponding to a small subset of the allowable moment pairs, can be modelled by it.

However, a pure empirical fitting of a model to the inverse Weibull data can lead to wrong model, and its effect on the estimated characteristics, such as the mean residual life $\mathrm{MRL}_{R}$ (6), can be quite severe. For example, Figure 4 shows that although the Cdfs of two models (inverse Weibull and log-logistic fitted to the same data) are quite close to each other, their $\mathrm{MRL}_{R}$ (6) functions are rather different. So, 
despite the fact the two models are both fitted to the same data and appear very close in Figure 4, the effect on critical prognoses of the misspecification is remarkable.

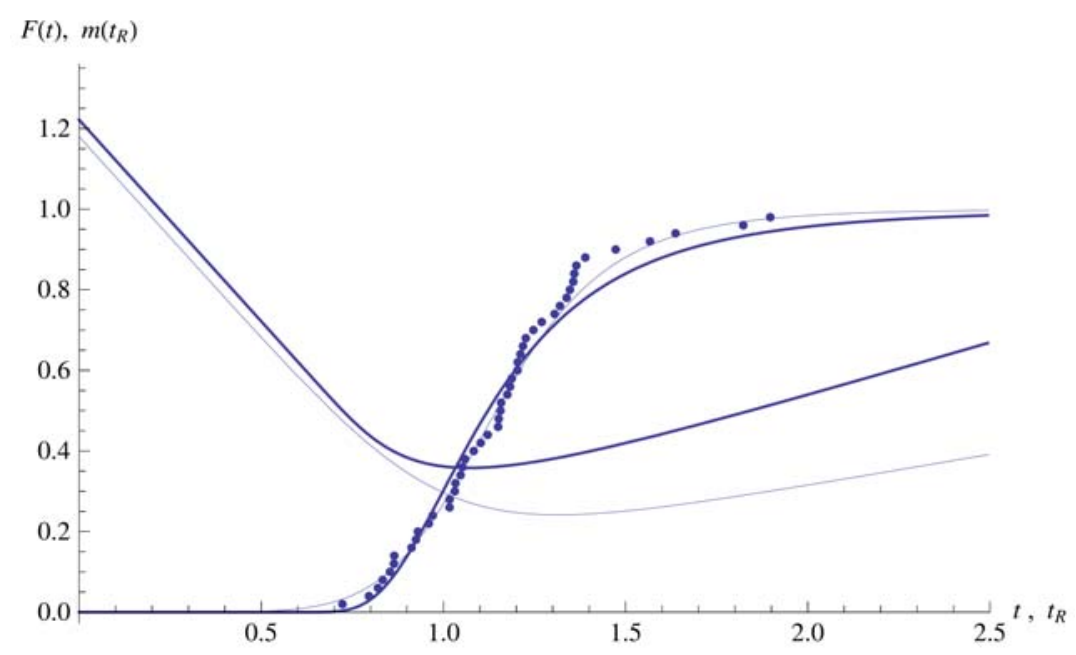

Figure 4. Distribution function $F(t)$ (S-shaped) and $\mathrm{MRL}_{R} m\left(t_{R}\right)$ (bathtub-shaped) of the inverse Weibull (thick lines), log-logistic (thin lines) models fitted to the same pseudo random sample (from a parent inverse Weibull distribution).

\section{Estimates of the Correct Selection Probabilities P-AD and P-MLL}

It is well-known that comparisons among many survival distributions can be successfully made by using a goodness-of-fit statistic at its maximum likelihood value (e.g., see Glen \& Leemis [12]) or by considering the difference of the maximized log-likelihoods (MLLs) and choosing the distribution with the largest value. Incidentally, in this paper, we chose the Anderson-Darling goodness-of-fit test (AD) (Anderson \& Darling [2]; Stephens [27]) because it emphasizes the tails of the presumed (i.e., hypothetical) parent distribution. 
With reference to pseudo random samples from parent inverse Weibull distribution, we found interesting to estimate the probability of correct selection between the inverse Weibull and log-logistic models. The probability was estimated in terms of the fraction of times (P-AD) that the fitted inverse Weibull model has the smaller Anderson-Darling statistic $A_{n}^{2}$ and the fraction of times (P-MLL) that the fitted inverse Weibull model has the larger maximized log-likelihood (MLL). To this end, we first found that for the inverse Weibull and log-logistic distributions both indices $A_{n}^{2}$ and MLL are pivotal quantities, that is independent of the hypothetical distribution parameters (intended as "arbitrary but determined" values). Then, for every combination of values $a=(1,2,3), b=(1.1,2.1,3.1,4.1,5.1)$, and $n=(10,30,50)$, we generated 1000 pseudo random samples from the parent inverse Weibull distribution and computed P-AD, P-MLL and the fraction of times (P-AD\&MLL) that the fitted inverse Weibull model has both the smaller Anderson-Darling statistic $A_{n}^{2}$ and the larger MLL. Thanks to the pivotal property of the $A_{n}^{2}$ and MLL indices, the conducted simulations gave 15 nearly identical results for each $n$. So, we have been able to evaluate a very reliable estimate of the probability of correct model selection (Table 1) based on the three examined criteria respectively. It is evident that the selection of the fitted model based upon the larger MLL has the highest probability of being correct. Moreover, the correct selection based on AD implies the correct selection based on MLL but not vice-versa.

Table 1. Probability of correct model selection estimated by averaging 15000 simulated results

\begin{tabular}{|c|c|c|c|}
\hline$n$ & P-AD & P-MLL & P-AD\&MLL \\
\hline 10 & 0.60 & 0.78 & 0.78 \\
\hline 30 & 0.77 & 0.88 & 0.88 \\
\hline 50 & 0.85 & 0.93 & 0.93 \\
\hline
\end{tabular}


However, when the size of the available sample is less than 30 , selecting the model purely on the basis of the empirical distribution implies an high probability of wrong selection.

\section{Real World Applicative Examples}

In this section, we provide two applicative examples to demonstrate the use of the generative mechanisms analytically shown before. These well known examples are highly representative of the critical real-world situations in which only tiny data sets are available, and so the probability of failure of pure statistical selection criteria could be very high (Table 1). Moreover, even though they involve very simple scientific knowledge they are effective applications of the proposed approach.

\subsection{Survival times of treated patients with Squamous carcinoma}

The dataset consists of survival times (in days) of 11 male patients affected by squamous carcinoma in the oropharynx and subjected to radiation therapy (see Ebrahimi [8], p. 413):

167, 238, 296, 324, 351, 372, 374, 404, 541, 560, 943.

The data result from a clinical trial carried out by a Radiation Therapy Oncology Group. The degradation process can be interpreted as an example of the "stress-strength" mechanism presented in Subsection 3.2. In fact, patients show a physical weakening over time as a consequence of the considerable toxic effects of the radiation treatment. Therefore, they exhibit a decreasing vital strength that can be modelled by a generic power law (11). The corresponding aggressiveness of the carcinoma appears to be a time independent random variable. So, it can be reasonably fitted by a Weibull model independent of $t$ (12). In [8], the data are used to illustrate some nonparametric estimation methods and no specific distributional model is advanced. On the basis of the above considerations, we are in a position to hypothesize that the inverse Weibull model is appropriate. 
The maximum likelihood estimates of the inverse Weibull parameters are $\hat{a}=0.00329$ and $\hat{b}=2.44$; the $A_{n}^{2}$ is equal to 0.340 , with $p$-value 0.526. So, the hypothesis of the inverse Weibull model cannot be rejected, with a degree of belief higher than a pure "statistical confidence" being supported by a probabilistic/biological analysis.

\subsection{Survival times of skin grafts on burn patients}

Lawless ([18], p. 340) reported the following 11 survival times (in days) of poorly matched skin grafts on burn patients:

$11,13,15,15,17,21,26,26,29,40,43$.

The death of the skin graft is due to the immune system of the recipient that attacks the graft transplanted from donor. The mechanism is exactly the same of the attempts that the immune system repeatedly does (randomly and with decreasing efficacy and energy) to destroy infecting organisms such as bacteria and viruses.

This is an example of the "latent-defensive-attempts" mechanism as the one described in Subsection 3.3. In fact, the immune system attempts can be modelled by the Poisson law (16) with success probability that decreases with the time $t$, according to a generic power law decreasing function (15). Then, the hypothesis of the inverse Weibull model seems to have good reasons. However, we consider also the Weibull model because it is the one supposed in ([18], p. 339).

The maximum likelihood estimates of the inverse Weibull parameters are $\hat{a}=0.0582$ and $\hat{b}=2.68$; the MLL $=-39.9$; the $A_{n}^{2}=0.31$ (with $p$-value 0.776). The maximum likelihood estimates of the Weibull parameters are $\beta=2.45$ and $\alpha=26.4$; the MLL $=-40.6$; the $A_{n}^{2}=0.372$ (with $p$-value 0.450$)$. It would seem that both models can be adopted even if they lead to very different critical prognoses. However, thank to the above careful probabilistic/biological analysis (and the comparison by means of the MLLs) the impasse can be overcame and the choice of the inverse Weibull model can be reasonably preferred. 


\section{Concluding Remarks}

- Although it is not always possible to achieve a closed-form expression of the distribution from a probabilistic generative mechanism (Lu \& Meeker [22]), the inverse Weibull distribution is obtained from the proposed three models of degenerative phenomena exactly. The knowledge of these models can also be exploited to perform the "probabilistic degradation analysis" needed to asses the hazard rate model when few or even no failure or injury data are expected to be observed (Lu \& Meeker [22]).

- The comparison with the log-logistic model is shown. The loglogistic model has been considered because: (a) it is the closest model which shares the upside-down bathtub (UBT) shaped hazard function; (b) it plays the role of a "frontier" (see Figure 3) separating the inverse Weibull model from many other alternative models on the $\left(\gamma_{2}, \gamma_{3}\right)$ chart from (Glen [11]; Vargo et al. [28]); (c) it gives a clear example of a model that - even though very well fitted to inverse Weibull data - may be very misleading because it entails highly incorrect assessments concerning, for instance, the mean residual life.

- The paper evaluates the probability of selecting the right model between the inverse Weibull and the log-logistic by choosing that one which minimizes the Anderson-Darling statistic or maximizes the likelihood. The paper shows that the two criteria have probabilities of correct selection that are respectively greater than 0.85 and 0.93 when the size of the available sample is greater than 50. Instead, when the size of the available sample is less than 30 (i.e., in a very frequent situation in the technological and biological fields) selecting the correct model purely on the basis of the empirical distribution remains a highly risky procedure, because the probabilities of wrong selection are respectively greater than 0.23 and 0.12 . In these cases, the careful analysis of the generative mechanisms of the involved random variable can be a decisive factor. 


\section{References}

[1] O. O. Aalen, Modelling heterogeneity in survival analysis by the compound Poisson distribution, The Annals of Applied Probability 2 (1992), 951-972.

[2] T. W. Anderson and D. A. Darling, A test of goodness-of-fit, Journal of the American Statistical Association 49 (1954), 765-769.

[3] R. D. Baker, Data-based modeling of the failure rate of repairable equipment, Lifetime Data Anal. 7 (2001), 65-83.

[4] F. Ballani, D. Stoyan and S. Wolf, On two damage accumulation models and their size effects, Journal of Applied Probability 44 (2007), 99-114.

[5] L. Bian and N. Gebraeel, Computing and updating the first-passage time distribution for randomly evolving degradation signals, IIE Transactions 44 (2012), 974-987.

[6] W. H. Carter, G. L. Wampler and D. M. Stablein, Regression Analysis of Survival Data in Cancer Chemotherapy, Marcel Dekker, New York, 1983.

[7] A. Drapella, Complementary Weibull distribution: Unknown or just forgotten, Quality and Reliability Engineering International 9 (1993), 383-385.

[8] N. Ebrahimi, Estimation of two ordered mean residual lifetime functions, Biometrics 49 (1993), 409-417.

[9] N. Z. Gebraeel, M. A. Lawley, R. Li and J. K. Ryan, Residual-life distributions from component degradation signals: A Bayesian approach, IIE Transactions 37 (2007), 543-557.

[10] R. E. Glaser, Bathtub and related failure rate characterizations, Journal of the American Statistical Association 75 (1980), 667-672.

[11] A. G. Glen, On the inverse gamma as survival distribution, Journal of Quality Technology 43 (2011), 158-166.

[12] A. G. Glen and L. Leemis, The arctangent survival distribution, Journal of Quality Technology 29 (1997), 205-210.

[13] C. N. Haas, Importance of distributional form in characterizing inputs to Monte Carlo risk assessments, Risk Analysis 17 (1997), 107-113.

[14] D. G. Harlow, Applications of the Fréchet distribution function, Int. Journal of Materials and Product Technology 17 (2002), 482-495.

[15] N. L. Johnson, S. Kotz and N. Balakrishnan, Continuous Univariate Distributions, Vol. 1, Wiley, New York, 1994.

[16] N. L. Johnson, S. Kotz and N. Balakrishnan, Continuous Univariate Distributions, Vol. 2, Wiley, New York, 1995.

[17] C. D. Lai and M. Xie, Stochastic Ageing and Dependence for Reliability, SpringerVerlag, New York, 2006. 
[18] J. F. Lawless, Statistical Models and Methods for Lifetime Data, Wiley, New York, 1982.

[19] L. Le Cam and J. Neyman, Probability Models and Cancer, North-Holland, Amsterdam, 1982.

[20] E. T. Lee, Statistical Methods for Survival Data Analysis, 2nd Edition, Wiley, New Jersey, 1992.

[21] M. Y. Lee and J. Tang, A modified em-algorithm for estimating the parameters of inverse Gaussian distribution based on time-censored Wiener degradation data, Statistica Sinica 17 (2007), 873-893.

[22] C. J. Lu and W. Q. Meeker, Using degradation measures to estimate a time-to failure distribution, Technometrics 35 (1993), 161-174.

[23] G. S. Mudholkar and G. D. Kollia, Generalized Weibull family: A structural analysis, Communications in Statistics - Theory and Methods 23 (1994), 1149-1171.

[24] D. N. P. Murthy, M. Xie and R. Jiang, Weibull Models, Wiley, Hoboken, New Jersey, 2004.

[25] S. S. Olin, D. A. Neumann, J. A. Foran and G. J. Scarano, Topics in cancer risk assessment, Environmental Health Perspectives 105 (1997), 117-126.

[26] E. V. Slud and J. Suntornchost, Parametric survival densities from phase-type models, Lifetime Data Anal. 20 (2014), 459-480.

[27] M. A. Stephens, Edf statistics for goodness of fit and some comparisons, Journal of the American Statistical Association 69 (1974), 730-737.

[28] E. Vargo, R. Pasupathy and L. M. Leemis, Moment-ratio diagrams for univariate distributions, Journal of Quality Technology 42 (2010), 276-286.

[29] P. Vilmos and E. Kurucz, Insect immunity: Evolutionary roots of the mammalian innate immune system, Immunology Letters 62 (1998), 59-66. 